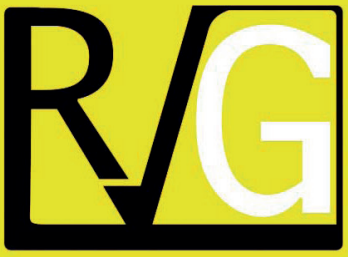

Julio - Septiembre, 2021

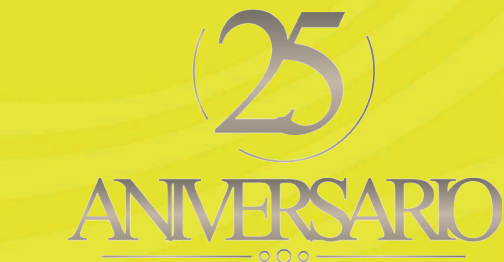

ANMERSARO
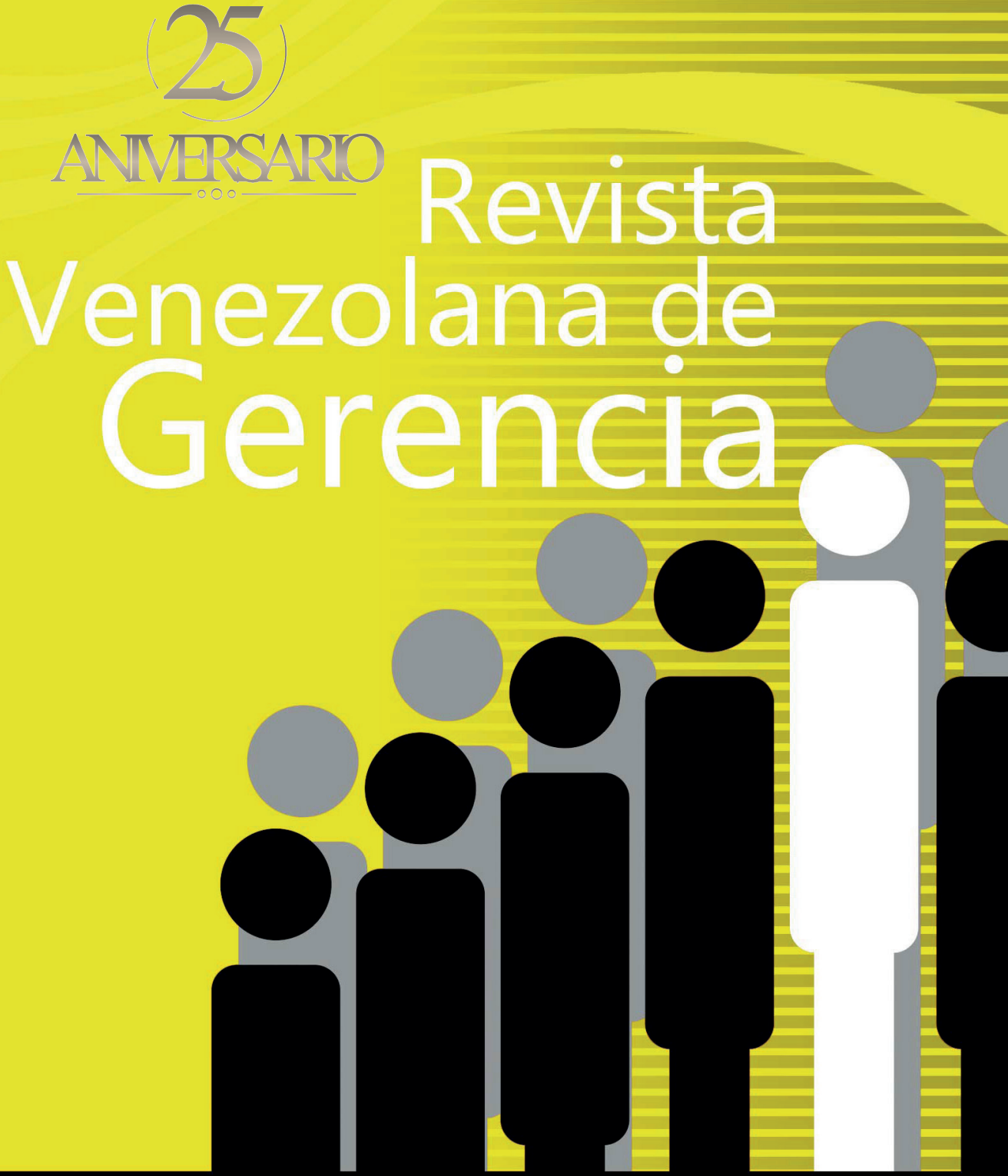

UNIVERSIDAD DEL ZULIA (LUZ)

Facultad de Ciencias Económicas y Sociales Centro de Estudios de la Empresa

ISSN 1315-99

Esta obra está bajo una licencia de Creative Comm Reconocimiento-NoComercial-Compartirlgual 3.0 Unpo http://creativecommons.org/licenses/by-nc-sa/3.0/deed.es 
COMO CITAR: Campos Trigoso, J., Murga Valderrama, N. L., y Rituay Trujillo, P. A., y García Rosero, L. M. (2021). Sostenibilidad del café: revisión sistemática de la literatura. Revista Venezolana de Gerencia, 26(95), 943-961. https://doi.org/10.52080/ rvgluz.27.95.30
Universidad del Zulia (LUZ)

Revista Venezolana de Gerencia (RVG)

Año 26 No. 95 Julio-Septiembre 2021, 943-961

ISSN 1315-9984 / e-ISSN 2477-9423

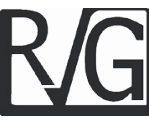

\title{
Sostenibilidad del café: revisión sistemática de la literatura
}

\author{
Campos Trigoso, Jonathan-Alberto* \\ Murga Valderrama, Nilton Luis** \\ Rituay Trujillo, Pablo-Alfredo*** \\ García Rosero, Ligia Magali****
}

\section{Resumen}

El objetivo de este trabajo fue analizar la evolución y las tendencias de investigación respecto a la evaluación de la sostenibilidad del café. Desde el plano metodológico, se realiza una revisión sistemática de la literatura durante el periodo comprendido entre los años 2010 al 2020. Se consideran artículos redactados en idioma inglés en tres bases de datos: Scopus, Science Direct y Springer Link, después de aplicar los criterios de inclusión y exclusión se analizaron 33 documentos, se encontró que existe una tendencia creciente en el estudio de la temática, los estudios son desarrollados principalmente en los países de Brasil, Vietnam, Uganda y Colombia; los principales medios de difusión son Sustainability (Switzerland), Ecological Economics, y Agricultural Systems. Se concluye que la evaluación de la sostenibilidad del café ha sido abordada principalmente a partir del marco de evaluación SAFA de la Organización de las Naciones Unidas para la Alimentación y la Agricultura (FAO), como método el de análisis de ciclo de vida ( $A C V$ ), y como técnica a la encuesta, demostrando además gran potencial para el estudio de esta temática la aplicación de Análisis Envolvente de Datos (DEA) y el Modelado Matemático Basado en Agentes (MPMAS).

Palabras clave: sostenibilidad; café; revisión sistemática.

Recibido: 04.03.21

Aceptado: 21.06.21

* Máster en Economía Agroalimentaria y del Medio Ambiente, Docente de la Escuela Profesional de Ingeniería en Agronegocios, Universidad Nacional Toribio Rodríguez de Mendoza de Amazonas, Perú. E-mail: jonathan. campos@untrm.edu.pe ORCID: https://orcid.org/0000-0002-4605-6005

** Maestro en Producción Animal, Docente de la Facultad de Ingeniería Zootecnista, Agronegocios y Biotecnología de la Universidad Nacional Toribio Rodríguez de Mendoza de Amazonas, Perú. E-mail: nmurga.fizab@untrm.edu.pe ORCID: https://orcid.org/0000-0002-1473-9055

*** Ingeniero en Agronegocios, Investigador del Instituto de Investigación en Negocios Agropecuarios, Universidad Nacional Toribio Rodríguez de Mendoza de Amazonas, Perú. E-mail: pablo.rituay@untrm.edu. pe ORCID: https://orcid.org/0000-0003-4338-6057

**** PhD en Agricultura Sustentable, Docente de la Universidad Nacional Toribio Rodríguez de Mendoza de Amazonas, Perú. E-mail: ligia.garcia@untrm.edu.pe ORCID: https://orcid.org/0000-0001-7508-7516 


\title{
Sustainability of coffee: systematic review of the literature
}

\begin{abstract}
The objective of this work was to analyze the evolution and trends of research regarding the evaluation of the sustainability of coffee from a systematic review of the literature during the period between 2010 and 2020. The study considers articles written in English in three databases: Scopus, Science Direct and Springer Link, after applying the inclusion and exclusion criteria, 33 documents were analyzed, it was found that there is a growing trend in the study of the subject, the studies are developed mainly in the countries of Brazil, Vietnam, Uganda and Colombia; the main media are Sustainability (Switzerland), Ecological Economics, and Agricultural Systems. It is concluded that the evaluation of the sustainability of coffee has been approached mainly from the FAO SAFA evaluation framework, as a method of life cycle analysis (LCA), and as a survey technique, also demonstrating great potential for the study of this subject the application of Data Envelope Analysis (DEA) and the Mathematical Modeling Based on Agents (MPMAS).
\end{abstract}

Keywords: Sustainabilit; coffee, systematic review.

\section{Introducción}

Existen más de 100 especies de café en el mundo, sin embargo, el comercio internacional se practica casi en su totalidad en Arábica (Coffea arabica) que representa el $70 \%$ del consumo mundial y Robusta (Coffea canephora) que representa el $30 \%$ restante. La variación entre ellas no solo incluye las condiciones de cultivo, también aspectos físicos como tamaño, forma, color, peso, y composición (Dias et al. 2015; Hameed et al. 2020).

Alrededor de 25 millones de personas en el mundo se dedican al cultivo y producción de café $\mathrm{y}, 500$ millones de personas se ganan sustento directa o indirectamente del negocio y su comercialización. América Latina, África Oriental y Asia son los principales productores de café Arábica, mientras que África occidental y central, el sudeste asiático y Brasil son los productores de granos de café Robusta (Hameed et al. 2020).

En el Perú, el café es el principal producto agrícola de exportación, con un área cultivada de 387,421 hectáreas. El $85 \%$ del total de caficultores son pequeños; poseen entre una y cinco hectáreas a en producción, destacando que solo el $30 \%$ de ellos se encuentran asociados en cooperativas (Ministerio de Desarrollo Agrario y Riego [MINAGRI], 2018).

Lo anterior explica, el por qué la mayoría de caficultores presentan serias dificultades para acceder a bienes y servicios agrarios, impactando negativamente en su competitividad y capacidad para enfrentar los desafíos vinculados al cambio climático, ataque 
de plagas, nuevos competidores, entre otros que amenaza la sostenibilidad de este cultivo (MINAGRI, 2018).

A partir de los acuerdos firmados en la Cumbre de la Tierra (Río de Janeiro, 1992) materializados en la Agenda 21, que se acelera la publicación de herramientas, metodologías y marcos para la evaluación de la sostenibilidad de los agroecosistemas (García-Montes \& Arnanz Monreal, 2019; González et al. 2020; Tyler, 2008).

Diversas instituciones proponen métodos y marcos para la medir la sostenibilidad agraria, destacan: Marco para la Evaluación de Sostenibilidad de los Sistemas Agrícolas y Alimentarios - SAFA (FAO, 2014); Marco para la Evaluación de Sistemas de Manejo de recursos naturales incorporando Indicadores de Sostenibilidad - MESMIS (López-Ridaura et al. 2002); Marco de Evaluación de la Sostenibilidad de la Agricultura y el Medio Ambiente - SAFE (Häni et al. 2003) entre otros.

En la actualidad se discute aún las características de los sistemas alimentarios sostenibles y el papel de agricultores, productores de alimentos y consumidores en dichos sistemas para proveer una alimentación adecuada, segura, asequible y saludable, que satisfaga las necesidades nutricionales de la población, de forma social y culturalmente aceptable, y sin desproteger los recursos naturales $y$ el medio ambiente (MacDonald \& Reitmeier, 2017). En este sentido, investigaciones han reportado que los pequeños caficultores enfrentan los desafíos respecto a la sostenibilidad de su actividad productiva a partir de la ejecución de algunas prácticas individuales y conjuntas.

Destacan las prácticas agroecológicas desarrolladas por una cooperativa en el Sur de la India, la misma demuestra en la práctica que el enfoque agroecológico no solo conserva la biodiversidad presente en los sistemas agro productivos sino que además reduce la dependencia a insumos externos en los cooperantes (Lanka et al. 2017). Otros autores consideran que las certificaciones voluntarias son una estrategia que promueve la sostenibilidad e incrementa el valor del café (Biswas-Tortajada \& Biswas, 2015; Grabs et al. 2016), así por ejemplo la Certificación Minas Café en Brasil, promueve la sostenibilidad de las fincas productoras de café en el largo plazo (Castro et al. 2018; Lourenzani et al. 2019).

Del mismo modo en Colombia, a partir de la evaluación de cuatro esquemas de gestión y certificación en fincas cafetaleras: convencional, comercio justo, orgánico y Rainforest Alliance, encontraron que estas mejoran la sostenibilidad en el cultivo de café (Bravo-Monroy et al. 2016).

En este contexto, la evaluación de la sostenibilidad de los sistemas de producción agrícolas, a través de la aplicación de metodologías, marcos e indicadores facilita la observación de tendencias en el desarrollo de los sistemas productivos, y la posterior detección e identificación de puntos críticos, que permitan establecer causas y proponer soluciones para su sostenibilidad (Tonolli \& Ferrer, 2018)

A partir de lo anterior, el objetivo de este artículo de revisión de literatura fue presentar un panorama del estado actual y las tendencias de las publicaciones sobre evaluación de la sostenibilidad del café, para alcanzar este objetivo se realizó una búsqueda sistemática de la literatura y lo recomendado por (de Oliveira et al. 2021; González et al. 2020; 
Koutsos et al. 2019; Lähtinen et al. 2014; Rituay et al. 2021; Traxler et al. 2020).

Se revisó la literatura concerniente a la evaluación de la sostenibilidad del café; se desarrolló una investigación de carácter teórico, clasificada como estudio bibliográfico o revisión sistemática, que selecciona y evalúa aportes, analiza y sintetiza datos, e informa la evidencia, de tal manera que permite llegar a conclusiones razonablemente claras sobre lo que se conoce y aquello que no (Chen, 2016).

El protocolo de investigación consideró cuatro actividades concernientes a la planificación de la revisión (Identificación de la necesidad de revisión de la literatura, establecimiento de las preguntas de investigación, creación de la cadena de búsqueda, e identificación de las fuentes de datos) $y$ dos que enmarcan el desarrollo de la misma (selección de estudios primarios y, extracción y síntesis de la información), todas ellas se detallan a continuación.

\section{Sostenibilidad del cultivo de café: revisión y análisis de la literatura}

A continuación se indican aspectos esenciales para a comprensión del cultivo de café.

\subsection{Identificación de la necesidad de revisión de literatura}

En la actualidad son diversas las instituciones multinacionales que promueven el desarrollo sostenible (ONU, UNESCO, OCDE, FAO, entre otras) y que han contribuido a la aceptación a nivel global del término de sustentabilidad, sin embargo esta aceptación también se debe a su ambigüedad (Pérez et al. 2016), pues la mayoría coinciden en que se debe alcanzar la misma, pero no hay consenso en su significado, lo que dificulta entonces su comprensión y medición ( (Sarandón, 2002).

En el campo de la investigación se presentan una tipología de estrategias para la evaluación de la sostenibilidad, una primera en la que se listan indicadores de sustentabilidad (IS) como los propuestos por la Comisión Económica para América Latina y el Caribe (CEPAL), el Fondo Mundial para la Naturaleza (WWF, por sus siglas en inglés), la Asociación de Estados del Caribe (AEC) y la División de Desarrollo Sostenible de las Naciones Unidas (UNDSD, por sus siglas en inglés), sin embargo existe acuerdo en que no se puede disponer de un conjunto universal de indicadores que puedan utilizarse en cualquier escenario.

La segunda estrategia para abordar la evaluación de la sostenibilidad es la compuesta por los Índices de Sostenibilidad, los cuales agregan la información relevante para la sustentabilidad de un sistema productivo en un valor numérico, aquí encontramos trabajos como los de (Gomez-Limon \& Arriaza, 2011; Méndez et al. 2016; Sarandón et al. 2008). En general la obtención de índices de sustentabilidad presenta el inconveniente de ponderar las variables analizadas y reducirlas a una unidad de medida única (Masera et al. 1999), se critica además su simplicidad y la carencia de un marco de análisis sólido que facilite derivar indicadores en contextos distintos a los evaluados.

Por último, la tercera estrategia, 
plantea el desarrollo de marcos de evaluación de la sostenibilidad que representan un vínculo entre el concepto teórico y su aplicación práctica. Se han identificado diferentes marcos de evaluación en el contexto de manejo de recursos naturales que presentan una estructura que va de principios o atributos hacia indicadores; estos últimos se definen bajo dos enfoques: de arriba hacia abajo (top-down), en el que los indicadores son definidos por expertos, y de abajo arriba, donde se definen previo análisis del sistema productivo en estudio.

Destacan entre los marcos de evaluación de sostenibilidad: el Marco paralaEvaluación delManejoSustentable de Tierras (FESLM, por sus siglas en inglés) (Smyth \& Dumanski, 1993); el marco del Instituto Interamericano de Cooperación para la Agricultura (IICA ) (De Camino \& Müller, 1993), el Marco para la Evaluación de Sistemas de Manejo Incorporando Indicadores de Sustentabilidad (MESMIS) (Masera et al. 1999); el Marco para Evaluación de la Sostenibilidad Inducida (RISE, por sus siglas en inglés) (Grenz et al. 2009; Häni et al. 2003) y el marco de Evaluación de Sostenibilidad de Sistemas de Alimentación y Agricultura (SAFA, por sus siglas en inglés) (FAO, 2014).

Dado lo anterior, alcanzar la sostenibilidad de la producción de café involucra una relación en el corto, mediano y largo plazo entre caficultores y medio ambiente. Evaluar su sostenibilidad, implica construir un conjunto de indicadores para su medición y caracterización presentados en diversas propuestas de instituciones y autores internacionales. Esta investigación pretende revisar las metodologías, marcos y herramientas de evaluación de la sostenibilidad que se han aplicado en la producción de café.

\subsection{Establecimiento de las preguntas de investigación}

Con base en investigaciones previas y como resultado de una revisión inicial de bases de datos de prestigio por los autores, se establecieron cinco preguntas de investigación que buscan responder al objetivo de esta investigación: ¿Cómo ha evolucionado el interés por evaluar la sostenibilidad de café?; ¿Cuáles son las principales fuentes de información científica en el tema de evaluación de la sostenibilidad de café?; ¿Qué dimensiones de sostenibilidad son abordadas por los artículos revisados?; ¿Qué eslabón de la cadena de valor del café es el más abordado en la evaluación de la sostenibilidad?; ¿Qué marco, método o técnica es el más utilizado para la evaluación de la sostenibilidad del café?

\subsection{Creación de la cadena de búsqueda para la revisión}

Para la obtención de los artículos relacionados con la temática de estudio se creó la cadena de búsqueda, para esto se identificaron las palabras claves a partir de las cinco preguntas de investigación. Se establecieron dos secciones, la primera aborda el término café y caficultura; la segunda referida a evaluación de sustentabilidad, e indicadores de sostenibilidad.

La cadena de búsqueda fue resultado del agrupamiento de las palabras clave y el uso de los operadores lógicos AND y OR; teniendo el siguiente resultado: coffee OR Coffee growing) AND (Sustainability OR Assessment OR 
Indicators.

\subsection{Identificación de las fuentes de datos}

A partir de lo recomendado por (Brammer, Branicki, y Linnenluecke, 2020), para la recuperación de los manuscritos científicos que sirvieron de fuente en esta investigación, se utilizaron las bases de datos: Scopus, Science Direct y Springer Link.

\subsection{Selección de estudios primarios}

Siguiendo el protocolo de investigación y a partir de una búsqueda imparcial, se identificó los estudios primarios para dar respuesta a las preguntas de investigación. Se definieron algunos criterios de inclusión y exclusión, y se siguió los mismos durante la selección de los estudios primarios.

Criterios de inclusión: (Cl01) estudios en idioma inglés; (Cl02) estudios publicados durante el periodo comprendido entre enero del 2010 y octubre de 2020; (ClO3) estudios que contengan al menos dos palabras clave en el título o resumen; (Cl04) estudios relacionados a la evaluación de la sostenibilidad.

Criterios de exclusión: (CE01) estudios duplicados; (CE02) estudios que no se basen en la evaluación de la sostenibilidad del café.

Para seleccionar cada uno de los estudios primarios se realizó lo siguiente: a) Adaptar la cadena de búsqueda al motor de la fuente de datos seleccionada. b) Aplicar los criterios de inclusión $\mathrm{Cl} 01$ y Cl02. C) Aplicar el criterio de inclusión Cl03. D) Leer resumen, introducción y conclusiones, y aplicar el criterio de inclusión $\mathrm{Cl04}$ y los criterios de exclusión CE01 y CE02.

\subsection{Extracción y síntesis de la información}

Previo al proceso de extracción de la información, los estudios primarios fueron organizados en el gestor de referencias Mendeley Desktop v.1.19.4. Posteriormente se realizó el análisis de la información de cada uno de los artículos seleccionados. Lo anterior se llevó a cabo a través de una hoja de cálculo en la que se recopilaron: título, autor, año, objetivo, método, marco o herramienta de evaluación de sustentabilidad, tipo de manuscrito y título de la fuente de cada estudio primario. Para extraer y analizar la información de todos los artículos, se realizó lo siguiente: (1) La información fue extraída por el primer autor, y (2) por separado se verificó la información por los otros autores.

En función de lo anterior, los resultados indican al evaluar la sostenibilidad del cultivo de café, sobresale el trabajo de (Ssebunya et al. 2019) quienes aplican el Marco SAFA de la FAO y comparan la sostenibilidad de fincas certificadas en comercio justo, producción orgánica, y aquellas no certificadas en Uganda. Encontraron que la certificación está asociada con un mejor desempeño en sostenibilidad de las fincas cafeteras en pequeña escala y que la misma mejora el logro de los objetivos de gobernanza a través de su influencia en la organización del grupo y las capacidades colectivas, además presenta efectos positivos en otras dimensiones de la sostenibilidad.

En Perú se reportaron evaluaciones de sostenibilidad del cultivo de café: 
(Mamani \& Jara, 2016) analizaron tres subsistemas: producción, beneficio húmedo, y beneficio seco de café orgánico y convencional, aplicaron el método síntesis emergética y calcularon e interpretación de indicadores de sostenibilidad, siendo favorables en el caso del sistema de producción orgánico y desfavorables para el caso del sistema de producción convencional. Del mismo modo (Márquez et al. 2016), aplicaron el método de análisis multicriterio en fincas con proceso de certificación orgánica, encontraron que el sistema de producción orgánico alcanzó un mayor índice general ambiental que el logrado por el sistema convencional.

En esta sección se responden las preguntas de investigación a través de los resultados obtenidos en la revisión sistemática de la literatura, se organizan en función de las mismas y nos permiten conocer el panorama general de la evaluación de la sostenibilidad del café.

A partir de la revisión de la literatura, se recuperó un total de 33 documentos con arreglo a los criterios de inclusión y exclusión (28 corresponden a la base de datos Scopus, 3 a Science Direct y 2 a Springer Link). Luego del análisis cronológico, se observa en la figura 1 que existe un incremento reciente, considerable, en cuanto a la publicación de estudios relacionados con la evaluación de la sostenibilidad del café, puntualmente en los años 2017, 2018, 2019 y 2020 (gráfico 1) que concentran el mayor número de publicaciones sobre la temática de estudio (el $76 \%$ ), se advierte un creciente interés de la academia por la temática objeto de revisión.

\section{Gráfico 1 \\ Publicaciones en evaluación de la sostenibilidad de café: Distribución cronológica}

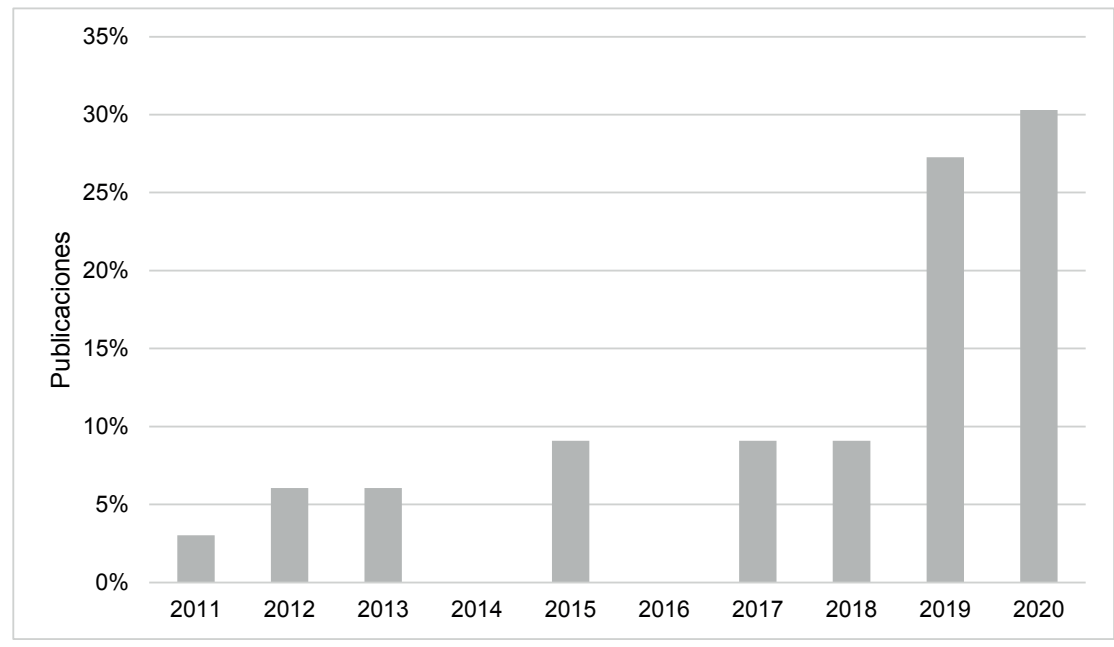


En cuanto, a los países que se encuentran desarrollando proyectos de investigación relacionadas con la evaluación de la sostenibilidad de café se encuentra, Brasil (21\%), Vietnam $(15 \%)$, Uganda $(9 \%)$ y Colombia $(9 \%)$ (Gráfico 2), destaca Brasil en materia de evaluación de la sostenibilidad ambiental de café convencional. De igual forma, De Muner et al. (2015) evaluaron la sostenibilidad bajo la perspectiva energética en tres sistemas de cultivo de café arábica; se enfrentó el café convencional, café de buenas prácticas agrícolas y café orgánico, siendo el más sostenible el café orgánico.

\section{Grafico 2}

\section{Distribución de publicaciones por países}

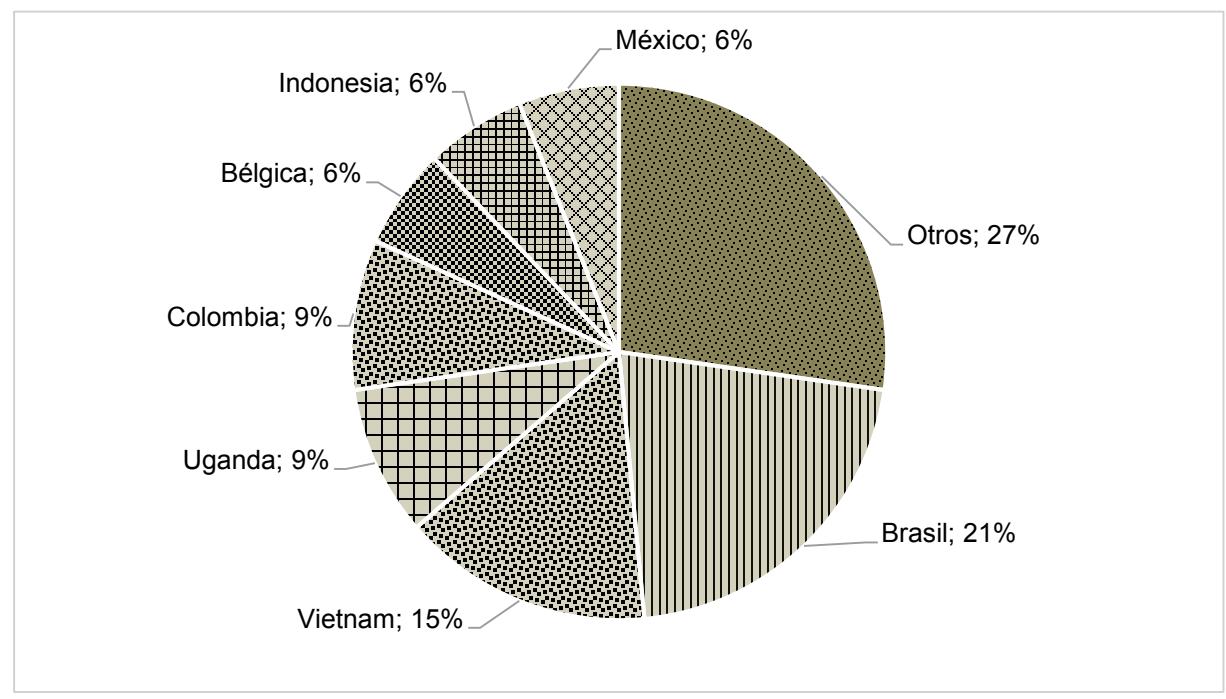

Fuente: Elaboración propia

Por su parte, Herzog et al. (2020) analizaron la sostenibilidad del café de variedad conilón en las región norte y sur de Espíritu Santo y la región de Bahía, aplicaron el cuestionario del programa público Agro+: por una agricultura más sostenible, logran un mejor índice de sostenibilidad la región norte de Espíritu Santo (55\%). En Vietnam (15\%), la investigación de (Trinh et al. 2020) evalúa la sostenibilidad de café a partir de la comparación del ciclo de vida en cultivos intensivos de café, cultivos moderados de café y cultivos orgánicos de café, siendo este último el que presenta menos impactos negativos en el medio ambiente y es más sostenible. Analizando Anh et al. (2019) el impacto del cultivo de café convencional y café sostenible, siendo el último más rentable, por lo que se recomienda su práctica para asegurar el desarrollo de la caficultura.

En relación con las principales 
fuentes de información científica en el tema de evaluación de la sostenibilidad de café, del total de estudios que componen la presente revisión sistemática, 32 fueron publicados en revistas y 1 corresponde a capítulo de libro.

En el gráfico 3, se muestran las revistas con mayor número de publicaciones durante el periodo de análisis de esta revisión en evaluación de la sostenibilidad de café. Destacan las revistas Sustainability (Switzerland) de la editorial MDPI, Ecological Economics, y Agricultural Systems, ambas de la editorial Elsevier, en estas revistas se publicaron el $21 \%$ de los estudios seleccionados en esta revisión. Sobresale el estudio de Manning et al. (2012) que aborda la cadena de valor global del café y su coevolución con los estándares voluntarios de sostenibilidad Comercio Justo, UTZ y el Código Común para la Comunidad Cafetera (4C), en esta investigación, los autores concluyen que son las condiciones económicas e institucionales en los países productores los impulsores para el desarrollo sostenible de la cadena de valor del café.

\section{Gráfico 3}

\section{Revistas en las cuáles se han publicado los artículos}

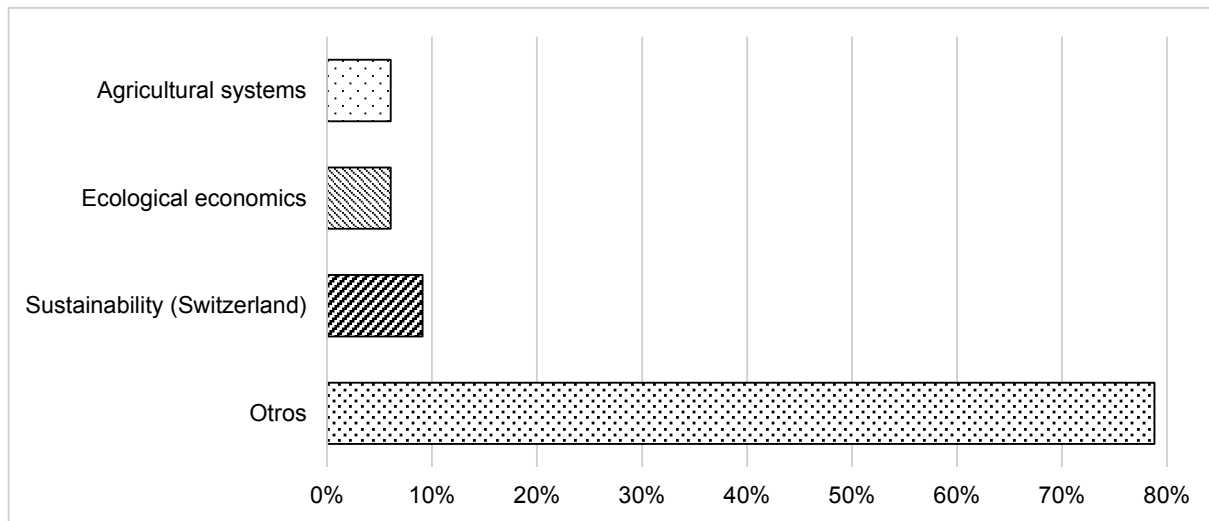

Fuente: Elaboración propia

De igual manera Acosta-Alba et al. (2019) proponen el marco LCA4CSA, el uso de la evaluación del ciclo de vida para fortalecer el análisis de sostenibilidad ambiental de las opciones de agricultura climáticamente inteligente a nivel de finca y sistema de cultivo, aplicando el mismo a la producción de café en Colombia. Se destaca que a partir de la práctica del uso de compost con residuos del procesamiento de café, se mejora entre un $22 \%$ y $41 \%$ la adaptación al cambio climático.

Siguiendo con el análisis 
bibliométrico, se muestran en el gráfico 4, las dimensiones de sostenibilidad abordadas en los artículos analizados. Se revela que los estudios primarios se concentran en primer lugar en la dimensión ambiental (6 artículos), los mismos aplican el método de ciclo de vida (Nab \& Maslin, 2020; Paula et al. 2020; Schmidt et al. 2020; Trinh et al. 2020) estimate and compare the environmental effects of conventional intensive, conventional moderate and organic intensive coffee cultivation methods in Vietnam. Life cycle assessment was used for the determination of environmental effects and carbon footprint for different coffee cultivation methods from cradle to gate. Functional unit was defined as $1 \mathrm{~kg}$ of green Robusta coffee bean in Tan Ha Commune, Lam Ha District, Lam Dong Province. The environmental effects of coffee cultivation were compared by SimaPro 8.3.0, and the two impact assessment methods used were IPCC 2013 v1.03 and $\mathrm{ReCiPe}$ v1.13. The life cycle assessment results in hotspot assessment for fertilizer and pesticide application showed that the conventional intensive contributed $85.5 \%$ to global warming owing to the high input of manure, whereas conventional moderate and organic intensive contributed $80.4 \%$ and $68 \%$ to global warming, respectively, throughout the 30 years of cultivation. Moreover, endpoint impact result indicated that human health is most affected by coffee cultivation compared to resources and ecosystem. The carbon footprint result of 1-year average productivity showed that the conventional intensive $(0.935 \mathrm{~kg}$ CO2e y la emergética (De Muner et al. 2015; Giannetti et al. 2011) para evaluar la sostenibilidad del cultivo de café.

En segundo lugar, la dimensión más evaluada en cuanto a la sostenibilidad es la económica (5 artículos), las mismas se realizaron a partir de la aplicación de encuestas y entrevistas (Do et al. 2019; Lingnau et al. 2019)having experience of over eight years in the field of various coffee industries in Vietnam, was created to give scores to multiple parameters. Technique for order of preference by similarity to ideal solution (TOPSIS, análisis económico de costo beneficio (Anh et al., 2019; Villavicencio-Enríquez, 2012), y simulación a partir de costos asociados a certificación (Latynskiy \& Berger, 2017). Los trabajos coinciden en que si bien es cierto las certificaciones sostenibles de café mejoran los beneficios o de los productores, estos no resultan significativos.

En tercer lugar, 2 artículos evalúan la dimensión social, analizan el impacto de las normas voluntarias de sostenibilidad en los medios de vida de los productores de café en Uganda (Chiputwa et al. 2015) y la sostenibilidad de productores de café certificados por terceros y los mecanismos de gobernanza en Brasil (Hajjar et al. 2019). Ambos estudios concluyen que las certificaciones mejoran los estándares de vida de los productores de café.

Por otro lado, 16 estudios (48\%) evalúan simultáneamente las tres dimensiones de la sostenibilidad; es de resaltar la investigación de (Winter et al. 2020)which represents an operationalization of the SAFA (Sustainability Assessment of Food and Agriculture systems quién evalúa el desempeño de sostenibilidad en café convencional y certificado en Brasil y Etiopía aplicando la herramienta SMARTfarm del marco de evaluación SAFA de la FAO. Sus hallazgos muestran que el café certificado muestra un mejor desempeño de sostenibilidad frente al convencional en ambos países, no obstante, el café 
convencional brasileño mostró un mejor desempeño en relaciones laborales y rentabilidad, esto último por la presencia de fincas con mayor extensión que permiten emplear personal permanente y beneficiarse de economías a escala.

Igualmente Almaraz et al. (2019) analizan la sostenibilidad en productores cafetaleros asociados y no en zonas marginadas de México, usando el marco MESMIS. Aplicaron encuestas a los productores, para posteriormente compararlos a través de ocho indicadores derivados de los atributos de sostenibilidad en los ejes: económico, social y ambiental; encontraron que los productores asociados a cooperativas presentan un índice de sostenibilidad mayor explicado principalmente por el sobreprecio del café y el acceso a innovaciones y capacitaciones permanente, concluyen que existe una relación entre cooperativas y sostenibilidad. Las cooperativas cafetaleras locales fortalecen el sector cafetalero y el desarrollo sostenible de la cadena productiva (gráfico 4).

\section{Gráfico 4}

\section{Distribución de estudios primarios por dimensión de sostenibilidad evaluada}

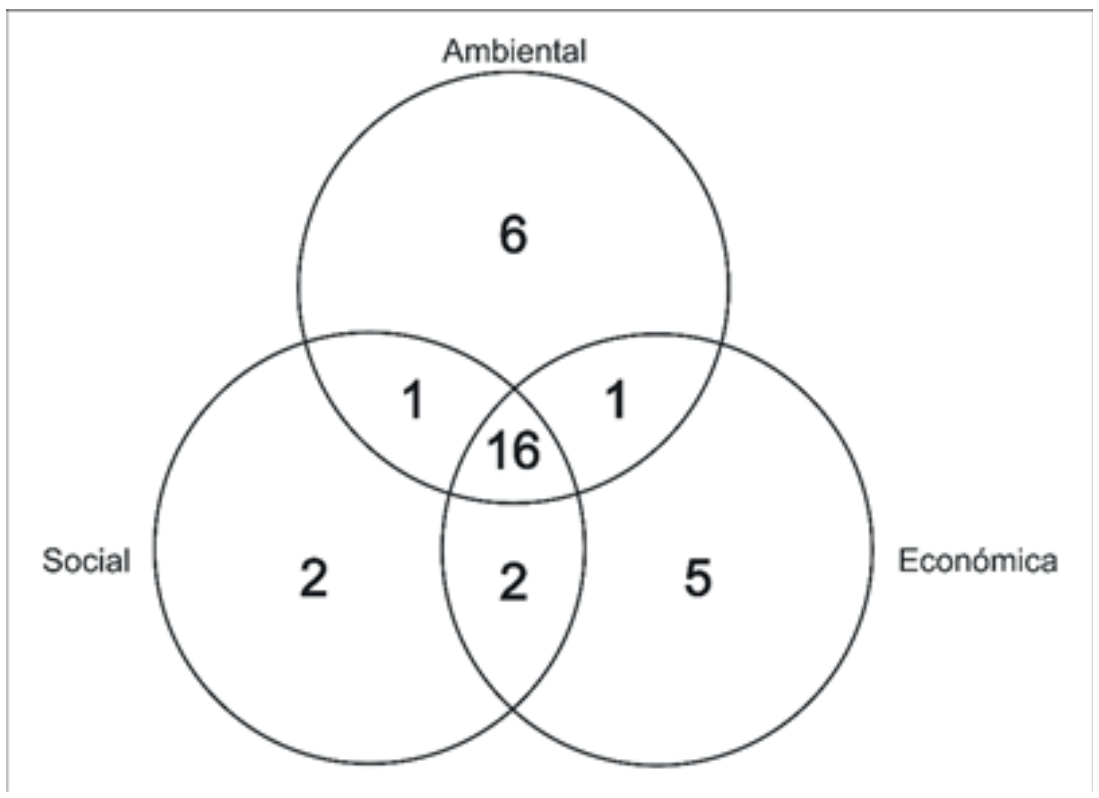

Fuente: Elaboración propia 
Otro aspecto que llama la atención busca responder a cuál de los eslabones de la cadena de valor del café es el más abordado en la evaluación de la sostenibilidad. En este sentido, los estudios se encuentran agrupados por el eslabón: producción, transformación, comercialización, o la cadena de valor en su totalidad, encontrándose 23 estudios $(70 \%)$ relacionados con el eslabón de producción; tanto café convencional como certificado sobresale las investigaciones de (Gaitán-Cremasch et al. 2018; Hamdan et al. 2019; Nab \& Maslin, 2020) quienes concuerdan en que las prácticas de producción sostenible son mejores para el medio ambiente $y$ debe fortalecerse la conservación de suelo y el uso de insumos óptimos para mejorar la rentabilidad y sostenibilidad de la producción de café, gráfico 5 .

\section{Gráfico 5}

\section{Distribución de estudios primarios según eslabón de la cadena de valor de café evaluada}

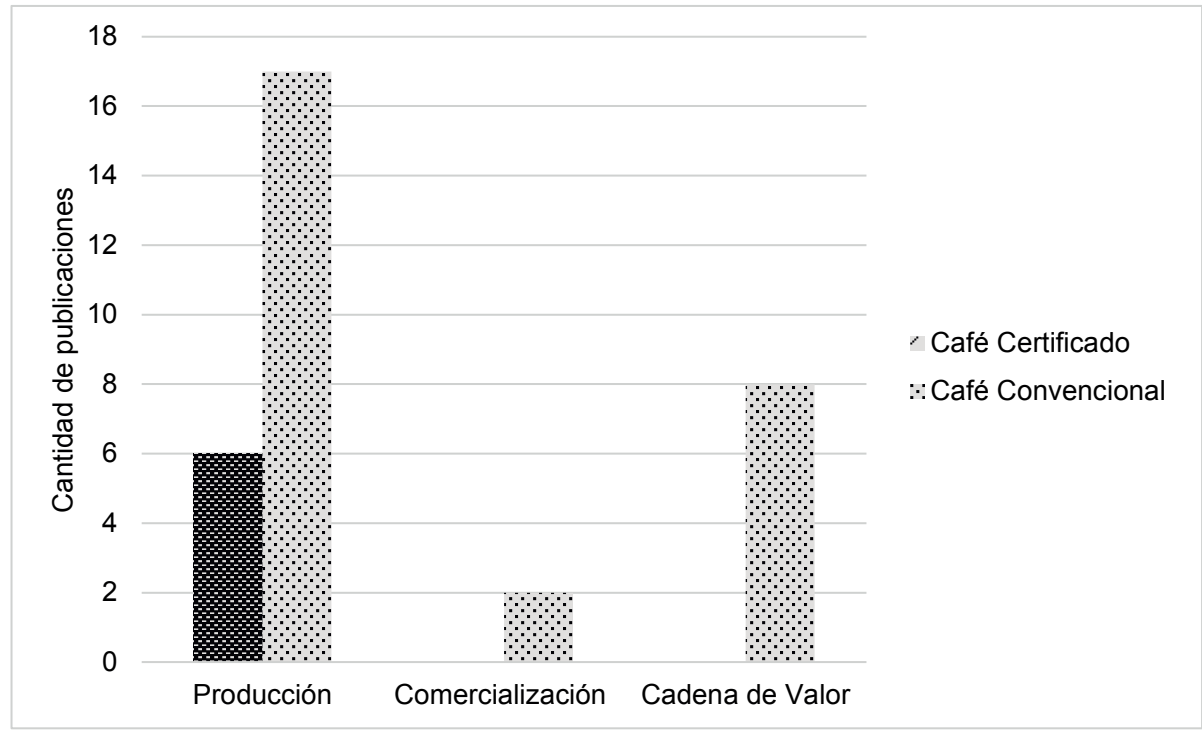

Fuente: Elaboración propia

Dos estudios se sitúan dentro del eslabón de comercialización, evaluaron el desempeño ambiental de la comercialización de café en presentaciones de cápsulas mono dosis (Vervaeke, 2012) y bajo el método tradicional, francés y aeropress (Paula et al. 2020) identificaron que la concentración de café, así como la proporción de masa de empaque por volumen de bebida preparada, tiene un efecto significativo sobre los 
impactos ambientales calculados y su sostenibilidad. Finalmente, ocho estudios evalúan toda la cadena de valor de café No se reportaron estudios en el eslabón de transformación.

Para finalizar, se indagó sobre el marco, método o técnica más utilizado para la evaluación de la sostenibilidad del café. Se precisan en la gráfico 6 los resultados respecto a las formas en las que los estudios abordaron la evaluación de la sostenibilidad de café, se observa que 7 (el 21\%) de estos utilizaron algún marco de evaluación de la sostenibilidad de los ya descritos en la sección dos de esta revisión, siendo el más aplicado SAFA de la FAO; 15 (el 45\%) utilizaron métodos, sobresale el cálculo de la sostenibilidad a través del análisis del ciclo de vida - ACV, por último 11 (el 33\%) aplicaron técnicas como la encuesta, entrevista y observación y la combinación de estas para evaluar la sostenibilidad en el café.

\section{Gráfico 6 \\ Marco, método o técnica utilizada en los estudios primarios}

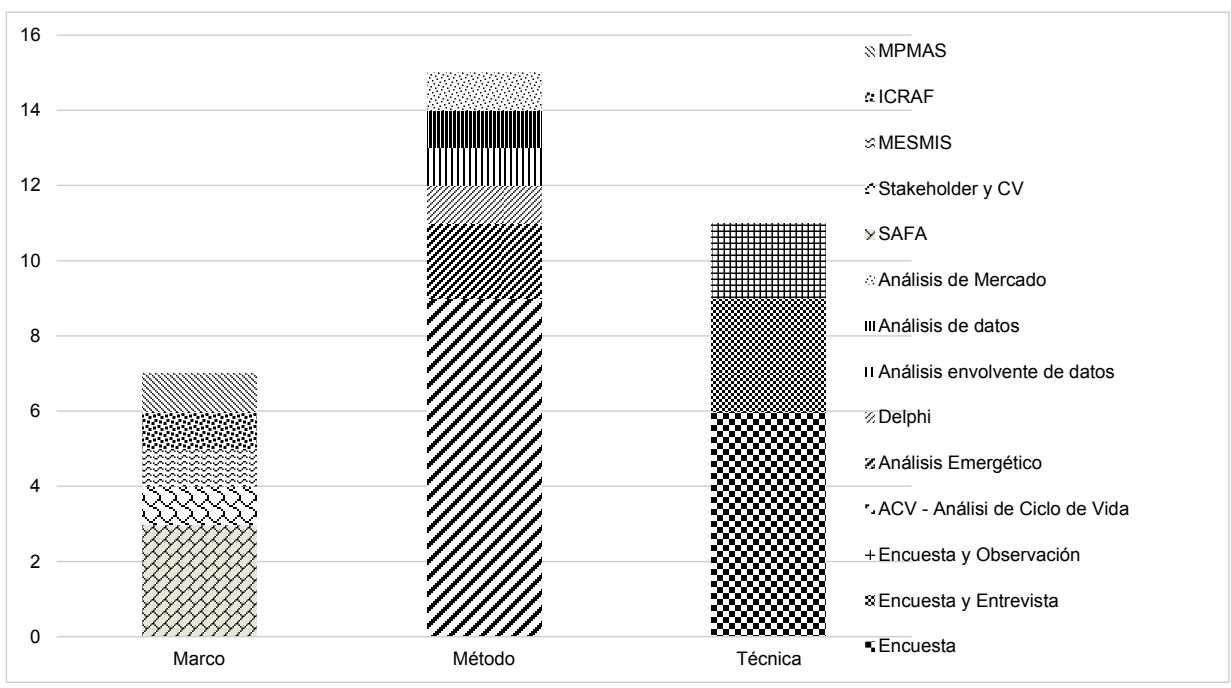

Fuente: Elaboración propia

\section{Conclusiones}

Los esfuerzos orientados a evaluar la sostenibilidad del café, como ha quedado revelado, requieren un enfoque holístico, coordinado y sinérgico que permita obtener conclusiones certeras respecto al estado, tendencias y transformaciones de la cadena de valor del café o de alguno de sus eslabones para formular estrategias que garanticen su desarrollo sostenible.

A través de los años emergen diversas formas de abordar la 
evaluación de la sostenibilidad del café, desde encuestas que permiten construir indicadores generales de sostenibilidad abordando los ejes económicos, ambientales y sociales, juntos o por separado; pasando por la aplicación de métodos, siendo el más usado el análisis del ciclo de vida (ACV); y marcos como el de Evaluación de Sostenibilidad de los Sistemas Agrícolas y Alimentarios (SAFA) de la FAO.

La revisión ha puesto en manifiesto que no existen indicadores, índices, técnicas, métodos, o marcos únicos para evaluar la sostenibilidad del cultivo de café, por el contrario, estos deben ser aplicados y construidos en realidades particulares y a partir de las mismas determinar su sostenibilidad. Se espera de esta manera despertar el interés para futuras investigaciones en esta temática.

Finalmente, esta investigación tiene 2 limitaciones principales: la primera es que se realizó la búsqueda en tres bases de datos: Scopus, Science Direct y Springer Link. La segunda es el hecho de que el estudio no considera las investigaciones publicadas en idiomas distintos al inglés. Sin embargo, se considera que la revisión presenta una muestra representativa de las investigaciones sobre sostenibilidad de café. Estudios futuros podrían incluir literatura en otros idiomas.

\section{Referencias bibliograficas}

Acosta-Alba, I., Chia, E., \& Andrieu, N. (2019). The LCA4CSA framework: Using life cycle assessment to strengthen environmental sustainability analysis of climate smart agriculture options at farm and crop system levels. Agricultural Systems, 171, 155-170. https://doi. org/10.1016/j.agsy.2019.02.001

Almaraz, V. P., Isabel, M., Rangel, P., Escoto, F. C., Ocampo, J., \& Aguilar, J. (2019). La asociación cooperativa como factor de sostenibilidad del sistema cafetalero en comunidades marginadas. REVESCO - Revista de Estudios Cooperativos, 131, 125-150. https://doi.org/10.5209/ REVE.63563.1.

Anh, N. H., Bokelmann, W., Nga, D. T., \& Minh, N. Van. (2019). Toward Sustainability or Efficiency: The Case of Smallholder Coffee Farmers in Vietnam. 2012(Mistiaen 2012), $1-25$.

Biswas-Tortajada, A., \& Biswas, A. K. (2015). Sustainability in Coffee Production. In Sustainability in Coffee Production: Creating Shared Value Chains in Colombia. Routledge. https://doi. org/10.4324/9781315697505

Brammer, S., Branicki, L., y Linnenluecke, M. K. (2020). COVID-19, Societalization, and the Future of Business in Society. Academy of Management Perspectives, 34(4). $\quad$ https://doi.org/10.5465/ amp.2019.0053

Bravo-Monroy, L., Potts, S. G., \& Tzanopoulos, J. (2016). Drivers influencing farmer decisions for adopting organic or conventional coffee management practices. Food Policy, 58, 49-61. https://doi. org/10.1016/j.foodpol.2015.11.003

Castro, C. V., Marcel, J., Lira, S., Salgado, E. G., \& Beijo, L. A. (2018). The continual improvement in the certification of coffee farms: a case study. Coffee Science, 13(4), 539549. http://www.coffeescience.ufla. br/index.php/Coffeescience/article/ view/1498

Chen, C. (2016). CiteSpace: A Practical 
Guide for Mapping Scientific Literature. Hauppauge, N.Y., Nova Science. https://novapublishers.com/ shop/citespace-a-practical-guide-formapping-scientific-literature/

Chiputwa, B., Spielman, D. J., \& Qaim, M. (2015). Food standards, certification, and poverty among coffee farmers in Uganda. World Development, 66, 400-412. https://doi.org/10.1016/j. worlddev.2014.09.006

De Camino, R., \& Müller, S. (1993). Sostenibilidad de la agricultura y los recursos naturales - Bases para establecer indicadores (IICA (ed.)).

De Muner, L. H., Masera, O., Fornazier, M. J., De Souza, C. V., \& De Loreto, M. D. D. S. (2015). Energetic sustainability of three arabica coffee growing systems used by family farming units in Espírito Santo state. Engenharia Agricola, 35(3), 397-405. https://doi.org/10.1590/1809-4430Eng.Agríc.v35n3p397-405/2015

de Oliveira, R. L. Z., de Oliveira, M. E., Trujillo, P. A. R., Camposo, J., \& Tech, A. R. B. (2021). Technologies applied in poultry production: A systematic review of the literature. RISTI - Revista Iberica de Sistemas e Tecnologias de Informacao, 2021(E39), 246-256.

Dias, F., Bugalho, M. N., RodríguezGonzález, P. M., Albuquerque, A., Orestes Cerdeira, J. (2015). Effects of forest certification on the ecological condition of Mediterranean streams. https://doi.org/10.1111/1365$\underline{2664.12358}$

Do, T. N., Kumar, V., \& Do, M. H. (2019). Prioritize the key parameters of Vietnamese coffee industries for sustainability. International Journal of Productivity and Performance Management, 69(6), 11531176. $\quad$ https://doi.org/10.1108/ IJPPM-06-2019-0282
Gaitán-Cremasch, D., Evert, F. K. Van, Jansen, D. M., Meuwissen, M., \& Oude Lansink, A. (2018). Assessing the Sustainability Performance of Coffee Farms in Vietnam: A Social Profit Inefficiency Approach. Sustainability (Switzerland), 10(4227). https://doi.org/10.3390/ su10114227

García-Montes, N., \& Arnanz Monreal, L. (2019). Metodologías participativas para la planificación de la sostenibilidad ambiental local. El caso de la Agenda 21. Empiria. Revista de Metodología de Ciencias Sociales, 44, 109-133. https://doi. org/10.5944/empiria.44.2019.25354

Giannetti, B. F., Ogura, Y., Bonilla, S H., \& Almeida, C. M. V. B. (2011). Emergy assessment of a coffee farm in Brazilian Cerrado considering in a broad form the environmental services , negative externalities and fair price. Agricultural Systems, 104(9), 679-688. https://doi. org/10.1016/j.agsy.2011.08.001

Gomez-Limon, J. A., \& Arriaza, M. (2011). Evaluación de la sostenibilidad de las explotaciones de olivar en Andalucía (Analistas Económicos de Andalucía (ed.); Issue October 2016).

González, A., Borrego, A., Villamil, D., \& Arteaga, W. (2020). Creación de valor sostenible: estudio documental sobre su origen y evolución. Revista Venezolana de Gerencia, 25(91), 780-795. https://doi.org/10.37960/ rvg.v25i91.33264

Grabs, J., Kilian, B., Hernández, D. C., \& Dietz, T. (2016). Understanding coffee certification dynamics: Aspatial analysis of voluntary sustainability standard proliferation. International Food and Agribusiness Management Review, 19(3), 31-56.

Grenz, J., Thalmann, C., Stämpfli, A., Struder, C., \& Häni, F. (2009). 
RISE - a method for assessing the sustainability of agricultural production at farm level. Rural Development News, 5-9. https:// saiplatform.org/uploads/Library/ RISEIndicatorsE RDN1 2009.pdf

Hajjar, R., Newton, P., Adshead, D., Bogaerts, M., Maguire-Rajpaul, V. A., Pinto, L. F. G., McDermott, C. L., Milder, J. C., Wollenberg, E., \& Agrawal, A. (2019). Scaling up sustainability in commodity agriculture: Transferability of governance mechanisms across the coffee and cattle sectors in Brazil. Journal of Cleaner Production, 206, 124-132. https://doi.org/10.1016/j. jclepro.2018.09.102

Hamdan, Fauzi, A. M., Rusli, M. S., \& Rustiadi, E. (2019). A study of the smallholder coffee agroindustry sustainability condition using the life cycle assessment approach in Bengkulu Province, Indonesia. Journal of Ecological Engineering, 20(6), 153-160. https://doi. org/10.12911/22998993/108637

Hameed, Z., Khan, I.U., Islam, T., Sheikh, Z. and Naeem, R.M. (2020), "Do green HRM practices influence employees' environmental performance?", International Journal of Manpower, 41(7), 1061-1079. https://doi.org/10.1108/IJM-08-20190407

Häni, F., Braga, F., Stämpfli, A., Keller, T., Fischer, M., \& Porsche, H. (2003). RISE , a Tool for Holistic Sustainability Assessment at the Farm Level. International Food and Agribusiness Management Review, 6(4).

Herzog, T. T., da Silva, M. B., \& Facco, A. G. (2020). Sustainabilit $y$ index in the production of conilon coffee. Revista Em Agronegocio e Meio Ambiente, 13(1), 213-232. https://doi.org/10.17765/2176-

\subsection{0v13n1p213-232}

Koutsos, T. M., Menexes, G. C., \& Dordas, C. A. (2019). An efficient framework for conducting systematic literature reviews in agricultural sciences. Science of the Total Environment, 682, 106-117. https://doi. org/10.1016/j.scitotenv.2019.04.354

Lähtinen, K., Myllyviita, T., Leskinen, P., \& Pitkänen, S. K. (2014). A systematic literature review on indicators to assess local sustainability of forest energy production. Renewable and Sustainable Energy Reviews, 40, 1202-1216. https://doi.org/10.1016/j. rser.2014.07.060

Lanka, S. V, Khadaroo, I., \& Böhm, S. (2017). Agroecology accounting: biodiversity and sustainable livelihoods from the margins. Accounting, Auditing and Accountability Journal, 30(7), 15921613. https://doi.org/10.1108/AAAJ12-2015-2363

Latynskiy, E., \& Berger, T. (2017). Assessing the Income Effects of Group Certification for Smallholder Coffee Farmers: Agent-based Simulation in Uganda. 68(3), 727748. https://doi.org/10.1111/1477$\underline{9552.12212}$

Lingnau, V., Fuchs, F., \& Beham, F. (2019). The impact of sustainability in coffee production on consumers' willingness to pay-new evidence from the field of ethical consumption. Journal of Management Control, 30(1), 65-93. https://doi.org/10.1007/ s00187-019-00276-X

López-Ridaura, $S$,, van Keulen, $H$., van Ittersum, M.K., y Leffelaar, P.A. (2005). Multi-scale Methodological Framework to Derive Criteria and Indicators for Sustainability Evaluation of Peasant Natural Resource Management Systems. Environment, Development and 
Sustainability, (7), 751-69. https:// link.springer.com/article/10.1007/ s10668-003-6976-x

Lourenzani, A. E. B. S., Watanabe, K., Pigatto, G. A. S., \& de Godoi Pereira, M. E. (2019). What fills your cup of coffee? The potential of geographical indication for family farmers' market access. In Coffee Consumption and Industry Strategies in Brazil: A Volume in the Consumer Science and Strategic Marketing Series. Elsevier Inc. https://doi.org/10.1016/ B978-0-12-814721-4.00014-7

MacDonald, R., y Reitmeier, Ch. (2017). Understanding Food Systems. Academic Press. https://www.elsevier.com/books/ understanding-food-systems/ macdonald/978-0-12-804445-2

Mamani, E. T., \& Jara, R. S. (2016). Sostenibilidad ambiental de dos sistemas de producción de café en Perú: orgánico y convencional. 6781, 51-65.

Manning, S., Boons, F., von Hagen, O., \& Reinecke, J. (2012). National contexts matter: The co-evolution of sustainability standards in global value chains. Ecological Economics, 83, 197-209. https://doi. org/10.1016/j.ecolecon.2011.08.029

Márquez, F., Julca, A., Manuel, C., Hugo, S., Silvana, V., \& Huerta, P. (2016). Sustentabilidad ambiental en fincas cafetaleras después de un proceso de certificación orgánica en la Convención (Cusco, Perú). Ecologia Aplicada, 2, 125-132. https://doi. org/http://dx.doi.org/10.21704/rea. v15i2.752

Masera, O., Astier, M., \& López-Ridaura, S. (1999). Sustentabilidad y manejo de recursos naturales: El marco de evaluación MESMIS (grupo interdisciplinario de tecnologia rural apropiada A.C (ed.)).
Méndez, M. S., Otiniano, A. J., Ventura, R. B., Nacional, U., \& La, A. (2016). Sustentabilidad de fincas productoras de café en jipijapa (manabí, ecuador). Saber y Hacer, 3(1), 23-35.

Ministerio de Desarrollo Agrario y Riego- MINAGRI (2018). Informe Diagnóstico ampliado del Sistema de Control Interno del Ministerio de Agricultura y Riego - Unidad Ejecutora 01 - Administración Central para el Período 2017 - 2018. https:// www.minagri.gob.pe/portal/actasdel-sistema-de-control-interno/actassci-2018

Nab, C., \& Maslin, M. (2020). Life cycle assessment synthesis of the carbon footprint of Arabica coffee: Case study of Brazil and Vietnam conventional and sustainable coffee production and export to the United Kingdom. https://doi.org/10.1002/ geo2.96

Naciones Unidas (1992). Declaración de Río sobre el Medio Ambiente y el Desarrollo. https://www.un.org/ spanish/esa/sustdev/documents/ declaracionrio.htm

Organización de las Naciones Unidas para la Alimentación y la AgriculturaFAO. (2014). Sustainability assessment of food and agriculture systems guidelines. http://www.fao. org/3/i3957e/i3957e.pdf

Paula, M., Tavares, D. F., \& Mourad, A. L. (2020). Coffee beverage preparation by different methods from an environmental perspective. Fao 2018.

Pérez, P., Pilar, M., Ávila, R., Eugenia, A., \& Torres, S. M. (2016). Evaluación de la sustentabilidad: una reflexión a partir del caso de la Red Nacional de Desarrollo Rural Sustentable ( México ) Sustainability evaluation : a reflection on the National Network for Sustainable Rural Development. 
Rituay, P., Murga Valderrama, N. L., Bustos Chavéz, M. D. P., Chauca Valqui, P., \& Campos Trigoso, J.A. (2021). Evolución y tendencias investigativas de tecnologías aplicadas en los agronegocios : una revisión sistemática de la literatura. Iberian Journal of Information Systems and Technologies, 189-199.

Sarandón, S. J. (2002). El desarrollo y uso de indicadores para evaluar la sustentabilidad de los agroecosistemas. Agroecología: El Camino Para Una Agricultura Sustentable, 393-414. http:// wp.ufpel.edu.br/consagrol files/2010/10/SARANDON-cap-20Sustentabilidad.pdf

Sarandón, S., Zuluaga, M. S., Cieza, R., Gómez, C., \& Janjetic, L. (2008). Evaluación de la sustentabilidad de sistemas agrícolas de fincas en misiones, argentina, mediante el uso de indicadores. Agroecología, 1, 19-28. https://revistas.um.es/ agroecologia/article/view/14

Schmidt, X. C., Gallego-schmid, A., \& Najdanovic-visak, V. (2020). Resources , Conservation \& Recycling Life cycle environmental sustainability of valorisation routes for spent coffee grounds: From waste to resources. Resources, Conservation \& Recycling, 157(February), 104751. https://doi.org/10.1016/i. resconrec. 2020.104751

Smyth, A. J., \& Dumanski, J. (1993). FESLM : An international framework for evaluating sustainable land management (Food and A).

Ssebunya, B. R., Schader, C., Baumgart, L., Landert, J., Altenbuchner, C., Schmid, E., \& Stolze, M. (2019). Sustainability Performance of Certified and Non-certified Smallholder Coffee Farms in Uganda. Ecological Economics,
156(August 2018), 35-47. https://doi. org/10.1016/j.ecolecon.2018.09.004

Tonolli, A. J., y Sergio Ferre, C. (2018). Comparison of agroecosystem assessment frameworks. Tropical and Subtropical Agroecosystems, 21(3), 487-504. https://www.revista. ccba.uady. $\mathrm{mx} /$ ojs/index.php/TSA/ article/view/2519/1187

Traxler, A. A., Schrack, D., \& Greiling, D. (2020). Sustainability reporting and management control - A systematic exploratory literature review. Journal of Cleaner Production, 276, 122725. https://doi.org/10.1016/j. jclepro.2020.122725

Trinh, L. T. K., Hu, A. H., Lan, Y. C., \& Chen, Z. H. (2020). Comparative life cycle assessment for conventional and organic coffee cultivation in Vietnam. International Journal of Environmental Science and Technology, 17(3), 1307-1324. https://doi.org/10.1007/s13762-01902539-5

Tyler Miller, G. (2008). Ciencia ambiental Desarrollo sostenible un enfoque integral. In Cengage Learning Latin America (Ed.), Innovación Educativa (8th ed., Issue 45).

Vervaeke, M. (2012). Life Cycle Assessment Software for Product and Process Sustainability Analysis. Chemical Education, 89, 884-890.

Villavicencio-Enríquez, L. (2012). Caracterización agroforestal en sistemas de café tradicional y rústico, en san miguel, veracruz, méxico. https://doi.org/10.5154/r. rchscfa.2010.08.051

Winter, E., Marton, S. M. R. R., Baumgart, L., Curran, M., Stolze, M., \& Schader, C. (2020). Evaluating the Sustainability Performance of Typical Conventional and Certified Coffee Production Systems in Brazil 
pp. 943-961

Revista Venezolana de Gerencia, Año 26 No. 95, 2021

and Ethiopia Based on Expert Judgements. Frontiers in Sustainable Food Systems, 4. https://doi. org/10.3389/fsufs. 2020.00049 